\title{
Dose-intense capecitabine, oxaliplatin and bevacizumab as first line treatment for metastatic, unresectable colorectal cancer: a multi-centre phase II study
}

\author{
Christopher GCA Jackson ${ }^{1}$, Katrina Sharples ${ }^{2,3}$, Paul I Thompson ${ }^{4}$, Anne O'Donnell ${ }^{5}$, Bridget Anne Robinson ${ }^{6}$, \\ David J Perez ${ }^{7}$, Jacqui Adams ${ }^{8}$, Richard Isaacs ${ }^{9}$, Sanjeev Deva ${ }^{3}$, Victoria A Hinder ${ }^{3}$ and Michael P Findlay ${ }^{3 *}$
}

\begin{abstract}
Background: Dose intense chemotherapy may improve efficacy with acceptable toxicity. A phase II study was conducted to determine the feasibility of a dose-intense two weekly schedule of capecitabine, oxaliplatin, and bevacizumab in metastatic colorectal cancer $(\mathrm{mCRC})$.

Methods: 49 patients with previously untreated $m C R C$ were recruited. Nineteen received capecitabine (1750 $\mathrm{mg} / \mathrm{m}^{2}$ oral BD days $\left.1-7\right)$ oxaliplatin $\left(85 \mathrm{mg} / \mathrm{m}^{2}\right.$ i.v. day 1$)$ and bevacizumab (5 mg/kg i.v. day 1$)$ using a 14-day cycle (C1750). Following toxicity concerns capecitabine was reduced to $1500 \mathrm{mg} / \mathrm{m}^{2}$ oral BD (C1500) and 30 further patients recruited.

Results: Over $80 \%$ of patients received at least $75 \%$ of planned chemotherapy doses over the first two cycles. At C1750 Grade 3 or higher toxicity occurred in $74 \%$ (95\% Cl 49\% to 91\%) and on C1500 in 70\% (95\% Cl 51\% to 85\%). The median progression-free survival was 6.9 months ( $95 \% \mathrm{Cl} 4.7$ to 8.7 ) for $\mathrm{C} 1750$ dose and 8.9 months ( $95 \% \mathrm{Cl} 4.1$ to 12.4) for C1500. 3 treatment-related deaths occurred.
\end{abstract}

Conclusions: Dose intense capecitabine and oxaliplatin with bevacizumab does not show additional efficacy and has potentially significant toxicity. Its use outside of clinical trials is not recommended.

Trial registration: ISRCTN41540878

Keywords: (6 in alphabetical order) bevacizumab, Capecitabine, Dose-intense, Colorectal cancer

\section{Background}

Colorectal cancer (CRC) is the second most incident solid tumour and the second leading cause of cancer-related death worldwide with over 1.2 million new cancer cases and 608,700 deaths estimated to have occurred in 2008 [1]. Due to an increasing incidence in developing countries as well as an aging population structure, the burden of colorectal cancer will continue to rise despite reductions in mortality in western countries [2]. With the use of chemotherapy and targeted agents for stage 4 disease, median overall survival has increased from 6 months with

\footnotetext{
* Correspondence: mp.findlay@auckland.ac.nz

${ }^{3}$ Cancer Trials New Zealand, University of Auckland, Level 1, Building 505, 85 Park Road, Grafton, Auckland, New Zealand

Full list of author information is available at the end of the article
}

supportive care alone to over 20 months. The monoclonal antibody against vascular endothelial growth factor, bevacizumab (Avastin, Roche), improved progression-free and over-all survival when combined with IFL chemotherapy [3], and increased progression-free survival with a nonstatistically significant increase in overall survival when combined with oxaliplatin based chemotherapy [4] and has been widely adapted as a standard component of first line therapy.

Capecitabine is an oral pro-drug of 5-fluorouracil, which in combination with Oxaliplatin (CapOx) has similar efficacy to the reference regimen FOLFOX-4, and obviates the need for a central venous access device and so is more convenient. Studies comparing CapOx in combination with bevacizumab have demonstrated similar 
PFS and overall survival to FOLFOX4-Bevacizumab [4,5]. However up to $20 \%$ of patients receiving CapOx experienced grade 3/4 diarrhoea [5-8] hence toxicity improvements in this schedule that maintain or improve efficacy are required.

The standard CapOx regimen is given on a 21-day cycle, with oxaliplatin $130 \mathrm{mg} / \mathrm{m}^{2}$ iv on Day 1 and capecitabine $1000 \mathrm{mg} / \mathrm{m}^{2}$ PO BD on days 1-14. Mathematical modelling as well as pre-clinical studies in breast cancer mouse xenografts have indicated that a dose-dense regimen of 7 days treatment with capecitabine followed by 7 days rest may result in a higher maximum tolerated dose being achieved [9]. Other groups have investigated the use of dose-dense capecitabine with oxaliplatin [10], adopting a two-weekly cycle with oxaliplatin $85 \mathrm{mg} / \mathrm{m}^{2}$ on Day 1, and a 7 day administration of capecitabine. In a dose escalation study using this $7 / 7$ schedule, a maximum tolerated dose of capecitabine was reached at $1750 \mathrm{mg} / \mathrm{m}^{2} \mathrm{PO}$ BDd1-7. The effective daily dose of capecitabine in a 21 day schedule is $1333 \mathrm{mg} / \mathrm{m}^{2} /$ day, whereas in the 14 day schedule using $1750 \mathrm{mg} / \mathrm{m}^{2}$ the daily dose received of capecitabine is $1750 / \mathrm{m}^{2} /$ day - a $30 \%$ increase in total capecitabine delivered.

In the follow-on phase II trial [11] 89 patients were randomised to receive either dose-intense CapOx or a standard 21 day regimen. Those allocated to the dose intense arm had a significantly longer median progressionfree survival time than those in the control arm $(10.5 \mathrm{v}$ 6.0 months; HR 2.15: 95\% CI 1.43 to $4.35 ; \mathrm{p}=0.0013)$. In addition, there were comparable rates of haematological and non-haematological toxicities, and only $12 \%$ Grade 3/4 diarrhoea observed in the dose-dense arm.

Given that the addition of bevacizumab to chemotherapy appears to improve PFS, and that dose-dense chemotherapy may improve efficacy, we considered that dose-intense CapOx with bevacizumab warranted exploration. We undertook a phase II study to determine the feasibility and safety of a dose-intense capecitabine and oxaliplatin schedule with bevacizumab for patients with previously untreated advanced colorectal cancer.

\section{Methods}

This national, multicentre, open-label, single arm, phase II clinical trial had the primary objective of determining the feasibility and safety of a dose-dense CapecitabineOxaliplatin-Bevacizumab regimen. Feasibility was determined by dose delivery, measured by the proportion of patients who received at least $75 \%$ of the planned dose for the first two cycles. Safety was measured according to Common Toxicity Criteria for Adverse Events v3.0. Secondary end-points included radiologic response rate, progression free survival and overall survival. The trial was approved by the Multi-Region Ethics Committee (MEC/ 06/04/041). The study was registered as ISRCTN41540878.

\section{Patient selection}

Patients were eligible if aged 18 years of age or older with previously untreated, histologically or cytologically confirmed locally recurrent or metastatic colorectal adenocarcinoma, ECOG performance status of 0 or 1 , absolute neutrophil count of $\geq 1.5 \times 10^{9} / \mathrm{L}$, platelet count of $\geq 75 \times 10^{9} / \mathrm{L}$, serum total bilirubin $<30 \mathrm{umol} / \mathrm{L}$, negative urinary protein on dipstick testing or $<1 \mathrm{~g} /$ 24 hour collection, and creatinine clearance $\geq 50 \mathrm{~mL} / \mathrm{min}$ by Cockcroft Gault calculation or direct measurement in accordance with local practice. All patients provided written informed consent. Prior adjuvant therapy was allowed if completed more than 6 months prior to enrollment. Exclusion criteria included prior chemotherapy for advanced $\mathrm{CRC}$, tumour invasion of major blood vessels, recent major surgery, clinically significant cerebrovascular or cardiovascular disease including uncontrolled hypertension, congenital or acquired coagulopathy or full anticoagulation prior to registration. Patients were enrolled from all 6 New Zealand Cancer Centres.

\section{Treatment}

Patients received capecitabine $1750 \mathrm{mg} / \mathrm{m}^{2}$ PO BD days $1-7$, oxaliplatin $85 \mathrm{mg} / \mathrm{m}^{2}$ i.v. day 1 and bevacizumab $5 \mathrm{~m} / \mathrm{kg}$ i.v. day 1 of a 14 day cycle (C1750). After an interim safety analysis the capecitabine dose was reduced to $1500 \mathrm{mg} / \mathrm{m}^{2}$ PO BD d1-7 (C1500) with doses of the other agents unchanged. Treatment was continued until disease progression, unacceptable toxicity or patient decision. All patients were followed for adverse events until 31st May 2008 and until 1 July 2009 for survival.

\section{Statistical considerations}

Dose delivery was assessed as the proportion of patients who received at least $75 \%$ of the planned chemotherapy dose over the first two cycles. Dose intensity for each patient was calculated as the average proportion of the per protocol dose given per day while still on study chemotherapy over the first two cycles. Toxicity was graded according to the National Cancer Institute Common Terminology Criteria for Adverse Events v 3.0 (NCICTCAE v3.0). Progression-free survival was calculated from day 1 of treatment until first documented evidence of disease progression or death from any cause. Modified RECIST 1.0 criteria were used to determine the tumour response, with confirmatory scans at 8 weeks (as opposed to 4) to reduce the burden of investigational procedures for the patients. For key outcome measures 95\% confidence intervals are reported for estimates of proportions. The Kaplan-Meier product limit estimator [12] was used to estimate progression free survival and overall survival. Median survival was reported, with confidence intervals calculated using the method of Brookemeyer and Crowley [13] with log transform. All analyses were stratified by 
planned capecitabine dose. A sample size of 60 patients was chosen to allow a precision of \pm 0.09 for a $95 \%$ confidence interval around an observed proportion of 0.8 .

\section{Results}

Patient characteristics

Between June 2006 and June 2007, 49 patients were recruited. Characteristics are summarized in Table 1. Nineteen patients received capecitabine at $1750 \mathrm{mg} / \mathrm{m} 2$ (C1750 group). An interim safety analysis was planned after 20 patients were recruited but was conducted after 19 patients due to investigator concerns about gastrointestinal toxicity. This resulted in a dose reduction in capecitabine to $1500 \mathrm{mg} / \mathrm{m} 2 / \mathrm{BD}$ (C1500 group), which was then received by 30 patients. Following 2 further deaths at this dose level, the study was terminated. The median follow-up was 23 months for the $\mathrm{C} 1750$ group and 15 months for the C1500 group.

\section{Dose delivery of chemotherapy}

Over $80 \%$ of patients received at least $75 \%$ of the planned dose of all three drugs in both dose groups (Table 2). The median dose-intensity for each drug over all cycles received was 0.8 for the C1750 group and was 0.96-1.0 in the $\mathrm{C} 1500$ group. Median number of cycles received was 7 for $\mathrm{C} 1750$ and 8 for $\mathrm{C} 1500$ (Figure 1, Table 2). The principal reasons for early discontinuation were gastrointestinal adverse events, fatigue, or pain. At the lower dose level, 15/30 patients discontinued treatment prior to progression. The main adverse events in this group were gastrointestinal, infection, sensory neuropathy and fatigue.

\section{Safety}

Among the 19 patients on C1750, 14 patients developed a grade 3 or greater toxicity (74\%; $95 \%$ CI $49-91 \%$ ); at C1500 grade $3 / 4$ toxicity occurred in 21 of 30 patients (70\%; 95\% CI 51-85\%) (Table 3). The most common toxicities at both dose levels were hand-foot syndrome, diarrhoea and nausea and vomiting. There were three treatment related deaths: one in the $\mathrm{C} 1750$ group due to an oesophageal perforation following chemotherapy induced emesis; two occurred in the C1500 group, with one patient developing fulminant diarrhea and subsequent sepsis 7 days after commencement of therapy, suggestive of DPD deficiency, and the other due to bowel perforation and sepsis in a patient with an unresected rectal primary. Following the third treatment-related death, the study was closed.

\section{Efficacy}

Forty-three patients were evaluable for response (Table 4), with two confirmed complete responses, and 20 partial responses (13 confirmed at 8 weeks). Of the seven unconfirmed responses, new lesions were found at the
Table 1 Patient demographic and disease characteristics

\begin{tabular}{|c|c|c|c|}
\hline & $\begin{array}{c}\text { Total } \\
n=49\end{array}$ & $\begin{array}{c}\mathrm{C} 1750 \mathrm{mg} / \mathrm{m}^{2} \\
\mathrm{n}=19\end{array}$ & $\begin{array}{c}\mathrm{C} 1500 \mathrm{mg} / \mathrm{m}^{2} \\
\mathrm{n}=30\end{array}$ \\
\hline $\begin{array}{l}\text { Age at registration, } \\
\text { median (IQ range) }\end{array}$ & $63(55,67)$ & $64(52,68)$ & $62(55,65)$ \\
\hline \multicolumn{4}{|l|}{ Gender, n (\%) } \\
\hline Female & $31(63.3)$ & $11(57.9)$ & $20(66.7)$ \\
\hline Male & $18(36.7)$ & $8(42.1)$ & $10(33.3)$ \\
\hline \multicolumn{4}{|l|}{ Ethnicity, n (\%) } \\
\hline European & $47(96.0)$ & $18(94.7)$ & $29(96.7)$ \\
\hline Maori & $1(2.0)$ & $1(5.3)$ & $0(0.0)$ \\
\hline Other & $1(2.0)$ & $0(0.0)$ & $1(3.3)$ \\
\hline \multicolumn{4}{|l|}{ Histology, n (\%) } \\
\hline Adenocarcinoma - NOS & $20(40.8)$ & $7(36.8)$ & $13(43.3)$ \\
\hline $\begin{array}{l}\text { Adenocarcinoma - poorly } \\
\text { differentiated }\end{array}$ & $4(8.2)$ & $2(10.5)$ & $2(6.7)$ \\
\hline $\begin{array}{l}\text { Adenocarcinoma - } \\
\text { moderately differentiated }\end{array}$ & $17(34.7)$ & $9(47.4)$ & $8(26.7)$ \\
\hline $\begin{array}{l}\text { Adenocarcinoma - well } \\
\text { differentiated }\end{array}$ & $1(2.0)$ & $1(5.3)$ & $0(0.0)$ \\
\hline Mucinious & $7(14.3)$ & $0(0.0)$ & $7(23.3)$ \\
\hline \multicolumn{4}{|l|}{ Disease Status, n (\%) } \\
\hline Distant metastases only & $41(83.7)$ & $17(89.5)$ & $24(80.0)$ \\
\hline Local recurrence & $5(10.2)$ & $2(10.5)$ & $3(10.0)$ \\
\hline $\begin{array}{l}\text { Distant metastases and } \\
\text { local recurrence }\end{array}$ & $3(6.1)$ & $0(0.0)$ & $3(10.0)$ \\
\hline \multicolumn{4}{|l|}{$\begin{array}{l}\text { Prior Anticancer } \\
\text { Treatment, n (\%) }\end{array}$} \\
\hline $\begin{array}{l}\text { Prior Adjuvant } \\
\text { Chemotherapy }\end{array}$ & $11(22.4)$ & $5(26.3)$ & $6(20.0)$ \\
\hline Radiotherapy & $8(16.3)$ & $3(15.8)$ & $5(16.7)$ \\
\hline Surgery & $44(89.8)$ & 17 (89.5) & $27(90.0)$ \\
\hline \multicolumn{4}{|l|}{$\begin{array}{l}\text { WHO Performance } \\
\text { Status, } \mathbf{n}(\%)\end{array}$} \\
\hline 0 & $42(85.7)$ & $18(94.7)$ & $24(80.0)$ \\
\hline 1 & $7(14.3)$ & $1(5.3)$ & $6(20.0)$ \\
\hline
\end{tabular}

confirmatory scan in three patients, one patient died, and the remaining three stopped treatment so no confirmatory scan was carried out. The response rates (confirmed) were $47.1 \%$ (95\% CI $23.0-72.2 \%$ ) for C1750 and 26.9\% (95\% CI 11.6-47.8) for C1500.

The median progression free and overall survival (Figures 2 and 3) for C1750 was 6.9 months (95\% CI 5.9 to 10.3 ) and 19.9 months (95\% CI 11.9-26.3), and for the C1500 group was 8.4 months (95\% CI 5.2 to 12.4 ) and 15.9 months (95\% CI 13.1-27.7).

\section{Discussion and conclusions}

Combination chemotherapy with capecitabine is a convenient alternative to infusional regimens with preserved 
Table 2 Compliance with chemotherapy

\begin{tabular}{|c|c|c|c|}
\hline & Total & $\begin{array}{l}\mathrm{C} 1750 \\
\mathrm{mg} / \mathrm{m}^{2}\end{array}$ & $\begin{array}{l}\mathrm{C} 1500 \\
\mathrm{mg} / \mathrm{m}^{2}\end{array}$ \\
\hline & $n=49$ & $n=19$ & $\mathrm{n}=30$ \\
\hline \multicolumn{4}{|c|}{$\begin{array}{l}\text { Reason for discontinuation } \\
\text { of chemotherapy, } n(\%)\end{array}$} \\
\hline Progression & $12(24.5)$ & $4(21.1)$ & $8(26.7)$ \\
\hline Need for surgery & $6(12.2)$ & $2(10.5)$ & $4(13.3)$ \\
\hline $\begin{array}{l}\text { Death (treatment-related } \\
\text { cause) }\end{array}$ & $3(6.1)$ & $1(5.3)$ & $2(6.7)$ \\
\hline Other toxicity & $16(32.7)$ & $5(26.3)$ & $11(36.7)$ \\
\hline $\begin{array}{l}\text { Patient or investigator } \\
\text { decision not otherwise } \\
\text { specified }\end{array}$ & $11(22.4)$ & $7(36.8)$ & $4(13.3)$ \\
\hline Still on treatment & $1(2.0)$ & $0(0.0)$ & $1(3.3)$ \\
\hline \multicolumn{4}{|l|}{$\begin{array}{l}\text { Receipt ofat least } 75 \% \\
\text { of planned dose in first } \\
\text { two cycles, } n(\%)\end{array}$} \\
\hline \multicolumn{4}{|l|}{ Each drug separately } \\
\hline Capecitabine & $42(85.7)$ & $18(94.7)$ & $24(80.0)$ \\
\hline Oxaliplatin & $46(93.9)$ & $19(100.0)$ & $27(90.0)$ \\
\hline Bevacizumab & $44(89.8)$ & $17(89.5)$ & $27(90.0)$ \\
\hline All three drugs & $40(82.6)$ & $16(84.0)$ & $24(80.0)$ \\
\hline \multicolumn{4}{|l|}{$\begin{array}{l}\text { Dose intensity while } \\
\text { on chemotherapy, } \\
\text { median (IQ range) }\end{array}$} \\
\hline Capecitabine & $0.95(0.66,1)$ & $0.8(0.57,1)$ & $0.96(0.67,1)$ \\
\hline Oxaliplatin & $1(0.8,1)$ & $0.8(0.67,1)$ & $1(1,1)$ \\
\hline Bevacizumab & $1(0.8,1)$ & $0.8(0.67,1)$ & $1(1,1)$ \\
\hline
\end{tabular}

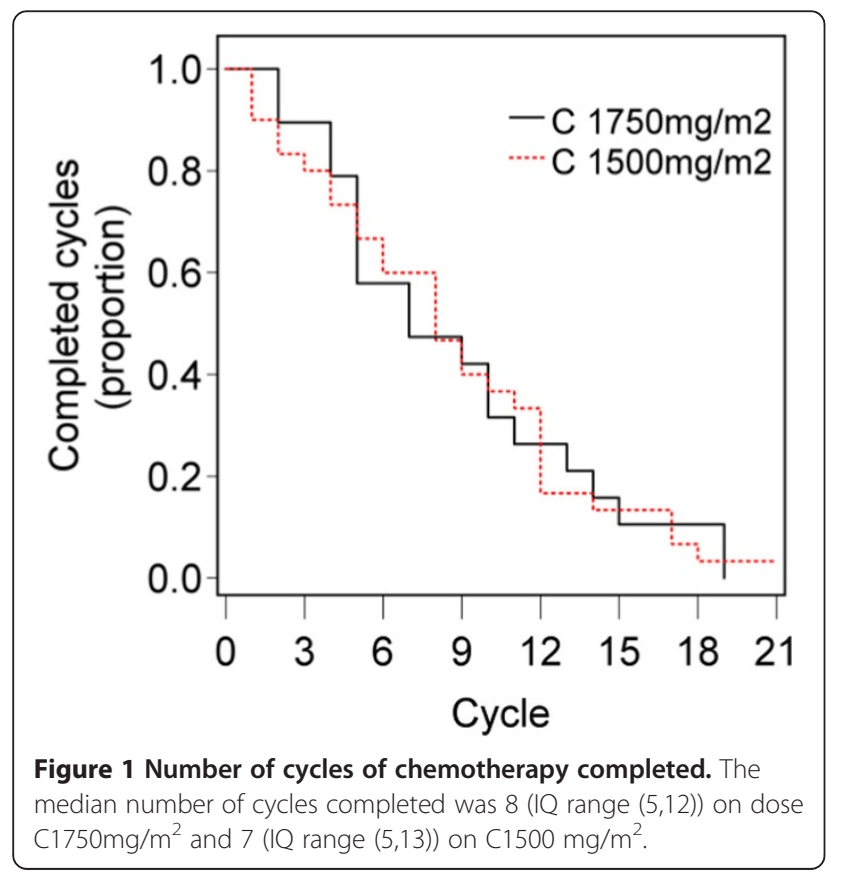

Table 3 Numbers of patients experiencing a grade 3 or higher adverse event up to $\mathbf{2 8}$ days after stopping chemotherapy

\begin{tabular}{|c|c|c|c|c|c|c|}
\hline \multirow[b]{3}{*}{$\begin{array}{l}\text { Hand-foot skin reaction } \\
\text { ( } \geq \text { grade } 2)\end{array}$} & \multirow{2}{*}{\multicolumn{2}{|c|}{$\begin{array}{c}\text { Total } \\
n=49\end{array}$}} & \multirow{2}{*}{\multicolumn{2}{|c|}{$\frac{\mathrm{C} 1750 \mathrm{mg} / \mathrm{m}^{2}}{\mathrm{n}=19}$}} & \multirow{2}{*}{\multicolumn{2}{|c|}{$\begin{array}{c}\mathrm{C} 1500 \mathrm{mg} / \mathrm{m}^{2} \\
\mathrm{n}=30\end{array}$}} \\
\hline & & & & & & \\
\hline & 14 & $(28.6)$ & 6 & (31.6) & 8 & $(26.7)$ \\
\hline Diarrhoea & 13 & $(26.5)$ & 6 & (31.6) & 7 & $(23.3)$ \\
\hline Nausea and vomiting & 9 & $(18.4)$ & 4 & $(21.1)$ & 5 & $(16.7)$ \\
\hline Pain - abdomen & 4 & $(8.2)$ & 2 & $(10.5)$ & 2 & (6.7) \\
\hline Perforation & 4 & (8.2) & 3 & $(15.8)$ & 1 & (3.3) \\
\hline Obstruction & 2 & (4.1) & 0 & $(0.0)$ & 2 & (6.7) \\
\hline Sensory neuropathy & 5 & $(10.2)$ & 2 & $(10.5)$ & 3 & $(10.0)$ \\
\hline Fatigue & 4 & (8.2) & 2 & $(10.5)$ & 2 & (6.7) \\
\hline $\begin{array}{l}\text { Thrombosis/thrombus/ } \\
\text { embolism }\end{array}$ & 4 & (8.2) & 2 & $(10.5)$ & 2 & (6.7) \\
\hline Hypokalemia & 4 & $(8.2)$ & 2 & $(10.5)$ & 2 & (6.7) \\
\hline Infection - sepsis & 3 & $(6.1)$ & 0 & $(0.0)$ & 3 & $(10.0)$ \\
\hline Febrile neutropenia & 2 & (4.1) & 1 & (5.3) & 1 & (3.3) \\
\hline ALT & 1 & (2.0) & 1 & (5.3) & 0 & $(0.0)$ \\
\hline Hypertension & 0 & $(0.0)$ & 0 & $(0.0)$ & 0 & $(0.0)$ \\
\hline $\begin{array}{l}\text { Acute respiratory distress } \\
\text { syndrome }\end{array}$ & 1 & (2.0) & 0 & $(0.0)$ & 1 & (3.3) \\
\hline $\begin{array}{l}\text { Any grade } 3 \text { or higher } \\
\text { adverse event }\end{array}$ & 35 & $(71.4)$ & 14 & (73.8) & 21 & $(70.0)$ \\
\hline 95\% confidence interval & $(56$. & $7,83.4)$ & & 90.9) & & $85.3)$ \\
\hline
\end{tabular}

efficacy, and dose-intense chemotherapy may result in greater dose delivery and enhanced efficacy over 21 day schedules. At the time of study inception, we were not aware of any published reports of a doseintense capecitabine-oxaliplatin schedule combined with bevacizumab.

Norton-Simon mathematical and pre-clinical models of capecitabine administration in breast cancer mouse xenografts have indicated that a 7 day treatment schedule followed by a seven day rest (7/7 schedule) may result in a higher maximum tolerated dose of capecitabine being achieved, with lower toxicity [9]. A phase 1 trial of capecitabine monotherapy in breast cancer found this $7 / 7$ schedule was well tolerated with $1 / 21$ patients experiencing grade 3 diarrhoea [14]. A subsequent phase 2 study of fixed-dose capecitabine $2000 \mathrm{mg} / \mathrm{PO} / \mathrm{BD}$ 7/7 combined with bevacizumab $10 \mathrm{mg} / \mathrm{kg}$ q14d had activity and reported $0 \%$ grade $3 / 4$ diarrhoea [15]. A randomised phase 2 study containing 89 patients compared standard CapOx (Cap1000 mg/m2/BD d1-14 q21d; Ox130 mg/m2 d1 q21d) with dose intense CapOx (Cap $1750 \mathrm{mg} / \mathrm{m} 2 / \mathrm{BD}$ $\mathrm{d} 1-7$, Ox85 mg/m2/d1; q14d) and reported a higher confirmed radiological response rate $(54.5 \% \mathrm{v} 42.2 \%)$ and longer PFS (10.5 v 6.0 months, $\mathrm{p}=0.0013$ ) favouring the dose intense schedule. Grade 3/4 diarrhoea rates were reported 
Table 4 Tumour response, progression and death

\begin{tabular}{|c|c|c|c|}
\hline & Total & $\begin{array}{l}\mathrm{C} 1750 \\
\mathrm{mg} / \mathrm{m}^{2}\end{array}$ & $\begin{array}{l}\mathrm{C} 1500 \\
\mathrm{mg} / \mathrm{m}^{2}\end{array}$ \\
\hline & $n=49$ & $\mathrm{n}=19$ & $\mathrm{n}=\mathbf{3 0}$ \\
\hline \multicolumn{4}{|l|}{$\begin{array}{l}\text { Patient status at end of } \\
\text { follow-up, } n(\%)\end{array}$} \\
\hline Alive, without disease progression & $9(18.4)$ & $1(5.3)$ & $8(26.7)$ \\
\hline Alive with disease progression & $19(38.8)$ & $9(47.4)$ & $10(33.3)$ \\
\hline Death after disease progression & $15(30.6)$ & $6(31.6)$ & $9(30.0)$ \\
\hline Death without disease progression & $6(12.2)$ & $3(15.8)$ & $3(10.0)$ \\
\hline Non measurable disease, $n$ & 6 & 2 & 4 \\
\hline Best overall response, $\mathrm{n}(\%)$ & $n=43$ & $n=17$ & $n=26$ \\
\hline Complete response & $2(4.7)$ & $1(5.9)$ & $1(3.9)$ \\
\hline Partial response & $20(46.5)$ & $8(47.1)$ & $12(46.2)$ \\
\hline Stable disease & $15(34.9)$ & $8(47.1)$ & $7(26.9)$ \\
\hline Progressive disease & $5(11.6)$ & $0(0.0 \%)$ & $5(19.2)$ \\
\hline Died & $1(2.3)$ & $0(0.0 \%)$ & $1(3.9)$ \\
\hline $\begin{array}{l}\text { Complete or partial response } \\
(\%, 95 \% \mathrm{Cl})\end{array}$ & $\begin{array}{c}51.2 \% \\
(35.5,66.7)\end{array}$ & $\begin{array}{c}52.9 \% \\
(27.8,77.0)\end{array}$ & $\begin{array}{c}50.0 \% \\
(29.9,70.0)\end{array}$ \\
\hline Confirmed response, $\mathrm{n}(\%)$ & $n=43$ & $n=17$ & $n=26$ \\
\hline Complete response & $2(4.7)$ & $1(5.9)$ & $1(3.9)$ \\
\hline Partial response & $13(30.2)$ & $7(41.2)$ & $6(23.1)$ \\
\hline Stable disease & $8(18.6)$ & $2(11.8)$ & $6(23.1)$ \\
\hline Progressive disease & $15(34.9)$ & $5(29.4)$ & $10(38.5)$ \\
\hline Died & $5(11.6)$ & $2(11.8)$ & $3(11.5)$ \\
\hline $\begin{array}{l}\text { Confirmed complete or partial } \\
\text { response }(\%, 95 \% \mathrm{Cl})\end{array}$ & $\begin{array}{c}34.9 \% \\
(21.0,50.9)\end{array}$ & $\begin{array}{c}47.1 \% \\
(23.0,72.2)\end{array}$ & $\begin{array}{c}26.9 \% \\
(11.6,47.8)\end{array}$ \\
\hline
\end{tabular}

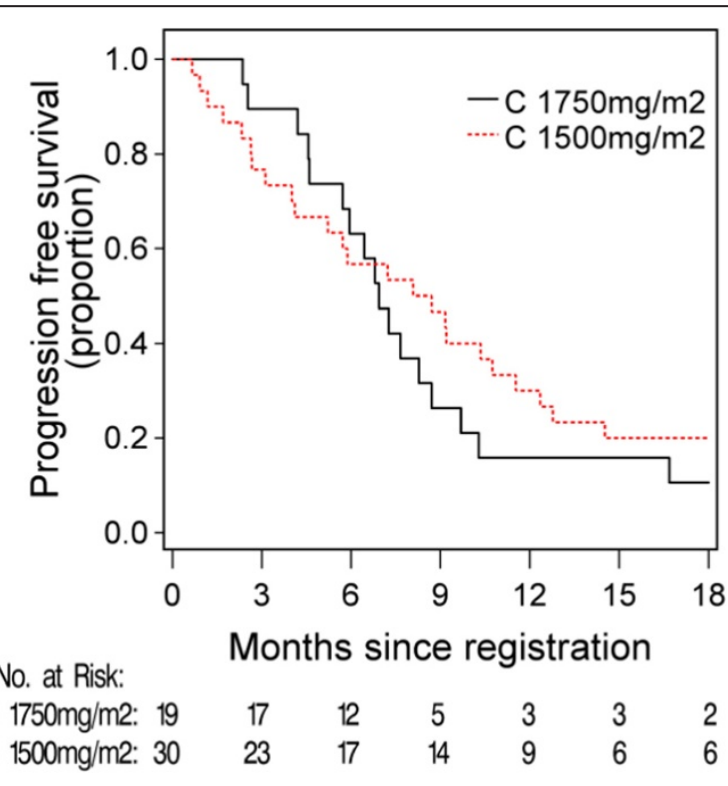

Figure 2 Kaplan-Meier estimates of progression free survival. The median progression-free survival was 6.9 months $(95 \% \mathrm{Cl}(5.9$, 10.3)) in the capecitabine $1750 \mathrm{mg} / \mathrm{m}^{2}$ group and 8.4 months ( $95 \%$ $\mathrm{Cl}(5.2,12.4))$ in the capecitabine $1500 \mathrm{mg} / \mathrm{m}^{2}$ group.

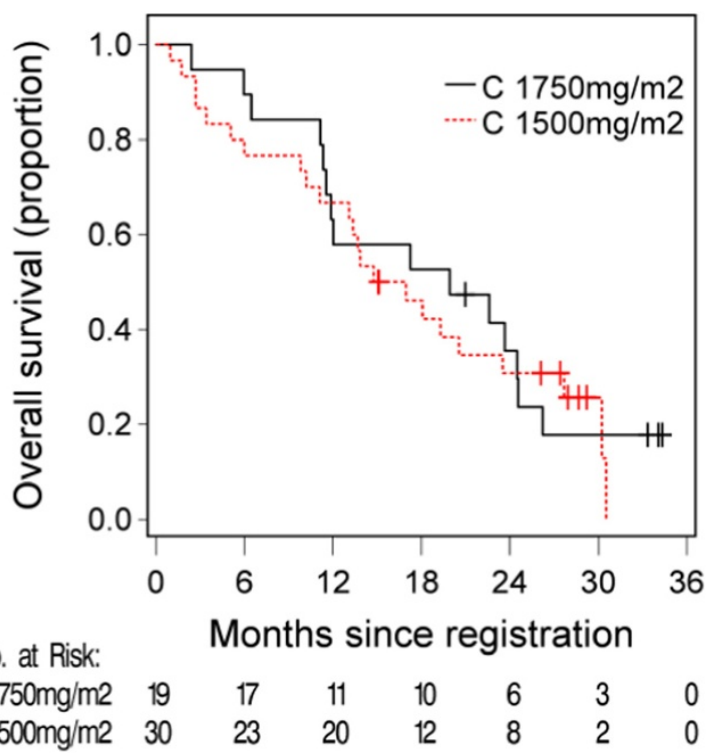

Figure $\mathbf{3}$ Kaplan-Meier estimates of overall survival. The median survival was 19.9 months $(95 \% \mathrm{Cl}(11.9,26.2))$ in the Capecitabine $1750 \mathrm{mg} / \mathrm{m}^{2}$ group and 15.9 months $(95 \% \mathrm{Cl}(13.1,27.7))$ in the capecitabine $1500 \mathrm{mg} / \mathrm{m}^{2}$ group.

as 9 and 12\% for the standard and dose intense arms respectively [11]. Reported rates of grade 3/4 diarrhoea of have been reported with three weekly CapOx-B regimens [16]. This contrasts with the results of a randomised phase 3 study, published following the completion of our study, of 435 patients of patients with advanced colorectal cancer, comparing 21 day Capecitabine with Oxaliplatin (Capecitabine $850 \mathrm{mg} / \mathrm{m} 2 / \mathrm{BD}$ d1-14 q21d; Oxaliplatin $130 \mathrm{mg} / \mathrm{m} 2 \mathrm{IV}$ d1 q21d) to a 14 day schedule with increased dose capecitabine (Cap $1500 \mathrm{mg} / \mathrm{m} 2 / \mathrm{BD}$ d1-7 q14d,Oxaliplatin $85 \mathrm{mg} / \mathrm{m} 2$ IV d1 q14d). This study showed the dose-intense regimen had a non-significantly shorter PFS compared to the standard regimen (8.4 months v 9.7 months; hazard ratio $[\mathrm{HR}]=0.84 ; 95 \% \mathrm{CI}=0.62$ 1.13). Patients in the dose intense group experienced higher rates of grade $3 / 4$ diarrhoea ( $29 \%$ vs $24 \%$ ), handfoot syndrome ( $12 \%$ vs $8 \%$ ), and treatment discontinuation rates (40\% vs 20\%) [17]. A summary of studies of doseintense regimens is described in Table 5.

Our study tested a dose of Capecitabine at the upper range of doses previously tested, with the addition of bevacizumab. Our observed rate of $23 \%$ G3/4 diarrhoea is comparable to other studies of Capecitabine-based doublets. Indeed our overall grade 3/4 adverse event rate of $74 \%$ is comparable to the $80 \%$ grade $3 / 4$ adverse event rate for FOLFOX4/CapOx-Bev arms seen in the NO16966 study [6]. However the 3 deaths represented unacceptably high toxicity, and we observed more perforations than seen in the NO16966 study. The overwhelming diarrhoea that resulted in the death of one patient happened after 
Table 5 Published studies of a dose-intense regimen of capecitabine and oxaliplatin

\begin{tabular}{|c|c|c|c|c|c|c|c|c|}
\hline \multirow[t]{2}{*}{ Trial } & \multirow[t]{2}{*}{ n } & \multirow{2}{*}{\multicolumn{2}{|c|}{ Chemotherapy }} & \multicolumn{2}{|c|}{ Relative dose intensity* } & \multirow{2}{*}{$\begin{array}{c}\text { Overall } \\
\text { response (\%) }\end{array}$} & \multirow{2}{*}{$\begin{array}{c}\text { PFS } \\
\text { (months) }\end{array}$} & \multirow{2}{*}{$\begin{array}{c}\mathrm{Gd} 3 / 4 \\
\text { diarrhoe } \\
(\%)\end{array}$} \\
\hline & & & & Ox & Cape & & & \\
\hline \multicolumn{9}{|l|}{ This trial } \\
\hline & & $\mathrm{q} 2 \mathrm{w}$ & Oxali $85 \mathrm{mg} / \mathrm{m}^{2}$ & & & & & \\
\hline & 19 & & Cape 1750 mg/m² BDd1-7 & $98 \%$ & $131 \%$ & $47.1 \%$ & 6.9 & $31.6 \%$ \\
\hline & 30 & & Cape $1500 \mathrm{mg} / \mathrm{m}^{2}$ BD d1-7 & $98 \%$ & $113 \%$ & $26.9 \%$ & 8.9 & $23.3 \%$ \\
\hline & & & Bevac 5 mg/kg & & & & & \\
\hline \multicolumn{9}{|l|}{ Phase II trials } \\
\hline \multirow[t]{5}{*}{ Scheithauer [11] } & 89 & $q 2 w$ & Oxali 85 mg/m² & $98 \%$ & $131 \%$ & $54.5 \%$ & 10.5 & $9 \%$ \\
\hline & & & Cape 1750 mg/m² BDd1-7 & & & & & \\
\hline & & & vs. & & & & & \\
\hline & & $\mathrm{q} 3 \mathrm{w}$ & Oxali $130 \mathrm{mg} / \mathrm{m}^{2}$ & & & $42.2 \%$ & 6 & $12 \%$ \\
\hline & & & Cape $1000 \mathrm{mg} / \mathrm{m}^{2}$ BD d1-14 & & & & & \\
\hline \multirow[t]{2}{*}{ Fedele [19] } & $47^{* *}$ & $\mathrm{q} 2 \mathrm{w}$ & Oxali $100 \mathrm{mg} / \mathrm{m}^{2}$ & $115 \%$ & $75 \%$ & $51 \%$ & - & $4.3 \%$ \\
\hline & & & Cape $1000 \mathrm{mg} / \mathrm{m}^{2} \mathrm{BD}$ d1 $1-7$ & & & & & \\
\hline \multirow[t]{3}{*}{ Yuan [20] } & $23^{* *}$ & $\mathrm{q} 2 \mathrm{w}$ & Oxali 85 mg/m² & $98 \%$ & N/A & $61 \%$ & - & $26 \%$ \\
\hline & & & Cape 2500 mg BD d1-7 & & & & & \\
\hline & & & Cetux 250 or $500 \mathrm{mg} / \mathrm{m}^{2}$ & & & & & \\
\hline \multirow[t]{4}{*}{ Lembersky [21] } & & $q 2 w$ & Oxali $85 \mathrm{mg} / \mathrm{m}^{2}$ & & & $38 \%$ & 10 & $18 \%$ \\
\hline & $11^{* * *}$ & & Cape $1250 \mathrm{mg} / \mathrm{m}^{2} \mathrm{BDd} 1-7$ & $98 \%$ & $94 \%$ & & & \\
\hline & 29 & & Cape 1500 mg/m² BDd1-7 & $98 \%$ & $113 \%$ & & & \\
\hline & & & Bevac 5 mg/kg & & & & & \\
\hline \multicolumn{9}{|l|}{ Phase III trials } \\
\hline \multirow[t]{7}{*}{ Hurwitz [17] } & 435 & $\mathrm{q} 2 \mathrm{w}$ & Oxali 85 mg/m² & $98 \%$ & $113 \%$ & $21.7 \%$ & 8.4 & $29 \%$ \\
\hline & & & Cape $1500 \mathrm{mg} / \mathrm{m}^{2}$ BD d1-7 & & & & & \\
\hline & & & Bevac 5 mg/kg & & & & & \\
\hline & & & vs. & & & & & \\
\hline & & $q 3 w$ & Oxali $130 \mathrm{mg} / \mathrm{m}^{2}$ & & & $29.4 \%$ & 9.7 & $24 \%$ \\
\hline & & & Cape $850 \mathrm{mg} / \mathrm{m}^{2}$ BD d1-7 & & & & & \\
\hline & & & Bevac 5 mg/kg & & & & & \\
\hline \multirow[t]{8}{*}{ Tournigand [22] } & 200 & $\mathrm{q} 2 \mathrm{w}$ & Oxali $100 \mathrm{mg} / \mathrm{m}^{2}$ & $115 \%$ & $94 \%$ & - & - & $21 \%$ \\
\hline & & & Cape $1250 \mathrm{mg} / \mathrm{m}^{2} \mathrm{BD}$ d1 $1-7$ & & & - & - & \\
\hline & & & Bevac 5 mg/kg & & & & & \\
\hline & & & vs. & & & & & \\
\hline & & $\mathrm{q} 2 \mathrm{w}$ & Oxali $100 \mathrm{mg} / \mathrm{m}^{2}$ & & & & & $5 \%$ \\
\hline & & & $\mathrm{LV} 400 \mathrm{mg} / \mathrm{m}^{2}$ & & & & & \\
\hline & & & $5 F U 2400 \mathrm{mg} / \mathrm{m}^{2}$ ci $46 \mathrm{hrs}$ & & & & & \\
\hline & & & Bevac 5 mg/kg & & & & & \\
\hline
\end{tabular}

Oxali: Oxaliplatin; Cape: Capecitabine; Bevac: Bevacizumab; LV: leucovorin; 5FU: 5-fluorouracil.

*Compared to standard CapeOx: oxaliplatin $130 \mathrm{mg} / \mathrm{m}^{2}$ on Day 1 and capecitabine $1000 \mathrm{mg} / \mathrm{m}^{2} / \mathrm{BD}$ D1-14.

**Number eligible for toxicity analysis.

***Dose of capecitabine was increased in trial due to tolerability at low dose.

7 days of therapy, suggesting that there may have been underlying DPD deficiency, and this death may not have been attributable to the dose-intense schedule. One of the deaths was from tumour site perforation that was deemed treatment-related. In the BEAT study, a phase 4 study of bevacizumab $5 \mathrm{mg} / \mathrm{kg}$ (biweekly regimens) or $7.5 \mathrm{mg} / \mathrm{kg}$ (3-weekly regimens) in combination with FOLFOX, CapOx (18\% of patients), FOLFIRI or 5-FU, 
tumour site perforation occurred in 3 of 223 patients with unresected colorectal cancers, indicating that this is a rare event [18]. The rates of perforation in our study were higher than seen in other studies. This may be either a chance finding or due to an interaction with this schedule. With these events our study could not demonstrate safety of the dose-intense CapOx-Bev regimen.

The response rate in the $\mathrm{C} 1750$ group was similar to other 5-FU-Oxaliplatin-Bevacizmab regimens and may have been higher if confirmatory scans were completed at 4 weeks instead of 8 . The response rate was lower at the reduced dose of capecitabine, however the study was not powered to compare the response rate between dose levels. The median PFS and OS of seen in our study are similar to other reported regimens. Our data are similar to the phase III trial published recently [17], with lower response rates than in the initial phase II studies, suggesting difficulty translating studies in more selected small groups of patients to the more general phase 3 population, even when performance status was relatively good. It is also noted that the eligible patients did not have resectable disease and were at the worse end of the spectrum for metastatic/recurrent disease.

The cluster of adverse events, particularly perforation and toxic death reminiscent of DPD deficiency may have been due to chance occurrence or may have been due to the toxicity of a dose intense regimen. A phase one design with a smaller population may not have detected these events, whereas a larger, randomised study may have balanced events between arms (if the adverse events are due to chance). These factors are limitations of a single-arm phase 2 design study.

The primary endpoint of the study was safety and feasibility as measured by the proportion of patients who received at least $75 \%$ of the planned dose for the first two cycles. Whilst this was achieved for $80 \%$ of participants, the toxicity over the course of treatment was too great. Despite preclinical modelling and two other studies of similar dose-intense regimens showing possible enhanced efficacy with acceptable toxicity, we could not demonstrate this with our regimen. We conclude that dose intense CapOx-Bev should not be used outside of clinical studies.

\section{Competing interests}

Roche New Zealand provided a grant to support core funding to Cancer Trials New Zealand separate to the funding of this study. MF is a Director of CTNZ. CJ received travel assistance to attend an educational meeting from Roche New Zealand. All remaining authors have declared no conflicts of interest.

\section{Authors' contributions}

Conception and design: MF KS. Provision of study materials or patients: MF KS PT AO BR DP JA RI VH. Collection and assembly of data: KS VH. Data analysis and interpretation: MF KS VH SD CJ. Manuscript writing: CJ SD MF. Final approval of manuscript: all authors.

\section{Acknowledgements}

This study was supported by a grant from Roche New Zealand.

Manufacturer name:

Capecitabine, Bevacizumab: Roche

Oxaliplatin: Sanofi Aventis

\section{Author details}

${ }^{1}$ Department of Medicine, University of Otago, Dunedin, New Zealand.

${ }^{2}$ Preventive and Social Medicine, University of Otago, Dunedin, New Zealand. ${ }^{3}$ Cancer Trials New Zealand, University of Auckland, Level 1, Building 505, 85 Park Road, Grafton, Auckland, New Zealand. ${ }^{4}$ Auckland City Hospital, Auckland, New Zealand. ${ }^{5}$ Wellington Cancer Centre, Capital and Coast Health, Wellington, New Zealand. ${ }^{6}$ Christchurch School of Medicine, University of Otago, Christchurch, New Zealand. ${ }^{7}$ University of Otago, Dunedin, New Zealand. ${ }^{8}$ Lyell McEwin Hospital, Elizabeth Vales, South Australia, Australia. ${ }^{9}$ Regional Cancer Treatment Service, MidCentral Health, Palmerston North, New Zealand.

Received: 5 March 2013 Accepted: 23 September 2014

Published: 2 October 2014

\section{References}

1. Jemal A, Bray F, Center MM, Ferlay J, Ward E, Forman D: Global cancer statistics. CA: A Cancer Journal for Clinicians 2011, 61(2):69-90.

2. Jemal A, Center MM, DeSantis C, Ward EM: Global patterns of cancer incidence and mortality rates and trends. Cancer Epidemiol Biomark Prev 2010, 19:1893-1907.

3. Hurwitz H, Fehrenbacher L, Novotny W, Cartwright T, Hainsworth J, Heim W, Berlin J, Baron A, Griffing S, Holmgren E, Ferrara N, Fyfe G, Rogers B, Ross R, Kabbinavar F: Bevacizumab plus irinotecan, fluorouracil, and leucovorin for metastatic colorectal cancer. N Engl J Med 2004, 350(23):2335-2342.

4. Saltz LB, Clarke S, Diaz-Rubio E, Scheithauer W, Figer A, Wong R, Koski S, Lichinitser M, Yang TS, Rivera F, Couture F, Sirzen F, Cassidy J: Bevacizumab in combination with oxaliplatin-based chemotherapy as first-line therapy in metastatic colorectal cancer: a randomized phase III study. Journal of clinical oncology: official journal of the American Society of Clinical Oncology 2008, 26(12):2013-2019.

5. Cassidy J, Clarke S, Diaz-Rubio E, Scheithauer W, Figer A, Wong R, Koski S, Lichinitser M, Yang TS, Rivera F, Couture F, Sirzen F, Saltz L: Randomized phase III study of capecitabine plus oxaliplatin compared with fluorouracil/folinic acid plus oxaliplatin as first-line therapy for metastatic colorectal cancer. J Clin Oncol 2008, 26(12):2006-2012.

6. Ducreux M, Bennouna J, Hebbar M, Ychou M, Lledo G, Conroy T, Adenis A, Faroux R, Rebischung C, Bergougnoux L, Kockler L, Douillard J-Y. Capecitabine plus oxaliplatin (XELOX) versus 5-fluorouracil/leucovorin plus oxaliplatin (FOLFOX-6) as first-line treatment for metastatic colorectal cancer. Int J Cancer 2011, 128:682-690.

7. Rothenberg ML, Cox JV, Butts C, Navarro M, Bang YJ, Goel R, Gollins S, Siu LL, Laguerre S, Cunningham D: Capecitabine plus oxaliplatin (XELOX) versus 5 -fluorouracil/folinic acid plus oxaliplatin (FOLFOX-4) as second-line therapy in metastatic colorectal cancer: a randomized phase III noninferiority study. Annals of oncology: official journal of the European Society for Medical Oncology / ESMO 2008, 19(10):1720-1726.

8. Cassidy J, Tabernero J, Twelves C, Brunet R, Butts C, Conroy T, Debraud F, Figer A, Grossmann J, Sawada N, Schoffski P, Sobrero A, Van Cutsem E, Diaz-Rubio E: XELOX (capecitabine plus oxaliplatin): active first-line therapy for patients with metastatic colorectal cancer. Journal of clinical oncology: official journal of the American Society of Clinical Oncology 2004, 22(11):2084-2091.

9. Norton L, Dugan U, Young D, Farrell C, Tanaka Y, Theodoulou M, Traina T, Hudis $\mathrm{C}$ : Optimizing chemotherapeutic dose-schedule (CDS) by Norton-Simon Modeling: Capecitabine (Xeloda $(\mathrm{R})=\mathrm{X})$. AACR Meeting Abstracts 2005, 2005(1):1182-d.

10. Scheithauer W, Kornek GV, Raderer M, Schull B, Schmid K, Langle F, Huber $\mathrm{H}$ : Intermittent weekly high-dose capecitabine in combination with oxaliplatin: a phase I/II study in first-line treatment of patients with advanced colorectal cancer. Ann Oncol 2002, 13:1583-1589.

11. Scheithauer W, Kornek GV, Raderer M, Schull B, Schmid K, Kovats E, Schneeweiss B, Lang F, Lenauer A, Depisch D: Randomized multicenter phase II trial of two different schedules of capecitabine plus oxaliplatin as first-line treatment in advanced colorectal cancer. J Clin Oncol 2003, 21:1307-1312 
12. Kaplan ELMP: Nonparametric estimation from incomplete observations. J Am Stat Assoc 1958, 53:457-481.

13. Brookmeyer RCJ: A confidence interval for the median survival time. Biometrics 1982, 38:29-41.

14. Traina TA, Theodoulou M, Feigin K, Patil S, Tan KL, Edwards C, Dugan U, Norton L, Hudis C: Phase I study of a novel capecitabine schedule based on the Norton-Simon mathematical model in patients with metastatic breast cancer. J Clin Oncol 2008, 26:1797-1802.

15. Gajiia D, Feigin K, Tan LK, Patil S, Geneus S, Theodoulou M, Norton L, Hudis CA, Traina TA: Phase 2 trial of a novel capecitabine dosing schedule in combination with bevacizumab for patients with metastatic breast cancer. Cancer 2011, 117:4125-4131.

16. Hochster HS, Hart LL, Ramanathan RK, Hainsworth JD, Griffing S, Mass RD, Nagarwala Y, Jirau-Lucca G, Shpilsky A, Childs BH: Safety and efficacy of oxaliplatin/fluoropyrimidine regimens with or without bevacizumab as first-line treatment of metastatic colorectal cancer ( $M C R C)$ : final analysis of the TREEStudy [abstract no. 3510], Presented at the 42nd Annual Meeting of the American Society of Clinical Oncology. Atlanta (GA): 2006 Jun 2-6.

17. Hurwitz H, Patt YZ, Henry D, Garbo L, Mitchell EP, Kohles J, Spigel D: Phase III study of standard triweekly versus dose-dense biweekly capecitabine (C) + Oxaliplatin (O) + Bevacizumab (B) as first-line treatment for metastatic colorectal cancer (mCRC): XELOX-a-DVS (dense versus standard): interim analysis. ASCO Meeting Abstracts 2009, 27:4078.

18. Van Cutsem E, Rivera F, Berry S, Kretzschmar A, Michael M, DiBartolomeo M, Mazier MA, Canon JL, Georgoulias V, Peeters M, Bridgewater J, Cunningham D, First BEAT investigators: Safety and efficacy of first-line bevacizumab with FOLFOX, XELOX, FOLFIRI and fluoropyrimidines in metastatic colorectal cancer: the BEAT study. Ann Oncol 2009, 20(11):1842-1847.

19. Fedele P, Di Maggio G, Leo S, Nanni F, Giuliani F, Biglietto M, Lorusso V, Cinieri S, Colucci G, Maiello E: Bi-weekly administration of capecitabine + oxaliplatin (Xelox-2) in first-line treatment of advanced colorectal cancer (ACRC): A phase II study of the Gruppo Oncologico dell'Italia Meridionale (GOIM). J Clin Oncol 2009, 27(suppl):abstr e15066.

20. Yuan Y, Ma H, Cohen D, Ryan T, Choi H, Love E, Awad M, Khambata-Ford S, Mauro D, Hochster H: Activity and tolerance of biweekly CapeOxcetuximab in 1st line therapy of metastatic colorectal cancer (mCRC): Relation to K-ras mutation status. J Clin Oncol 2009, 27(suppl):abstr e15018.

21. Lembersky B, Sehgal R, Rajasenan K, Crandall T, Balaban E, Pinkerton A, Kane P, Potter D, Kinney C, Ramanathan RK: Phase II study of bevacizumab (BEV), weekly (wkly) intermittent capecitabine (Cap), and oxaliplatin (Ox) on an every-2-week schedule for patients (Pts) with untreated advanced colorectal cancer (CRC). J Clin Oncol 2008, 26(suppl):abstr 4061.

22. Tournigand C, Lledo G, Delord J, André T, Maindrault-Goebel F, Louvet $C$, Scheithauer W, de Gramont A: Modified (m)Folfox7/bevacizumab (B) or modified $(m)$ Xelox/bevacizumab with or without erlotinib $(E)$ in first-line metastatic colorectal cancer (MCRC): Results of the feasibility phase of the DREAM-OPTIMOX3 study (GERCOR). J Clin Oncol 2007 25(18S):suppl; abstr 4097.

doi:10.1186/1471-2407-14-737

Cite this article as: Jackson et al:: Dose-intense capecitabine, oxaliplatin and bevacizumab as first line treatment for metastatic, unresectable colorectal cancer: a multi-centre phase II study. BMC Cancer 2014 14:737.

\section{Submit your next manuscript to BioMed Central and take full advantage of:}

- Convenient online submission

- Thorough peer review

- No space constraints or color figure charges

- Immediate publication on acceptance

- Inclusion in PubMed, CAS, Scopus and Google Scholar

- Research which is freely available for redistribution

Submit your manuscript at www.biomedcentral.com/submit
C Biomed Central 